\title{
TEACHER TRAINEES' ATTITUDES AND MOTIVATIONS TOWARDS LEARNING TE REO MĀORI
}

\author{
Russell S. Pine*
}

\begin{abstract}
A discussion about the decreasing proficiency levels of one of the official languages in New Zealand, te reo Māori, would not be complete without understanding teacher trainees' attitudes and motivations towards taking an optional Māori language course. This is because teacher trainees can provide significant opportunities within the classroom to promote learning of te reo and understanding their perspectives on learning the language to inform future revitalisation efforts. Therefore, the aim of the research reported here was to investigate trainees' attitudes about and motivations for enrolling in an optional Māori language course in order to provide a thematic overview and guide future research on te reo revitalisation efforts. The attitude and motivations of language learners have been shown to be significant determinants in identifying whether a minority language can succeed in a bilingual environment. The data collected from an online survey and a focus group were analysed to identify five distinct but also related themes that were commonly found across participants: (a) importance of engaging in culture alongside the language, (b) consistent learning throughout school and college, (c) motivation can be enhanced through everyday use of the language with friends and whānau, (d) large expenditure of time and effort is required to access Māori speaking communities and (e) mismatch between attitudes towards Māori language and behaviours. The results show how important understanding learners' attitudes and motivations are when considering the effectiveness of developing revitalisation interventions for te reo.
\end{abstract}

* Independent researcher, Victoria University of Wellington, Wellington, New Zealand.

Email: russell.pine@education.govt.nz

DOI: 10.20507/MAIJournal.2018.7.2.4 


\section{Keywords}

te reo Māori, tikanga, attitudes and motivations, New Zealand

Curriculum, learning languages, second language revitalisation

\section{Introduction}

In 1987, te reo Māori was finally accepted and recognised as one of the official languages of New Zealand (Te Puni Kōkiri, 2003). However, despite its recognition and value in society, the proportion of Māori who can hold an everyday conversation in te reo has continued to decrease over the years, from $24.97 \%$ in 1996 to $21.3 \%$ in 2013 (Statistics New Zealand, 2013). According to King (2001), attitudes and motivations play significant roles in determining whether a minority language can succeed in a bilingual or multilingual context. Furthermore, when interventions such as immersion programmes are being made to revitalise a language, motivations and attitudes held by speakers and society as a whole are crucial factors in determining whether the revitalisation efforts will succeed.

According to Eagly and Chaiken (1993), attitude can be defined as "a psychological tendency that is expressed by evaluating a particular entity with some degree of favor or disfavor" (p. 1). In simple terms, it can be viewed as a judgement summarising a collective ideology of ideas or opinions. In comparison, motivation underpins the individual's desire to act in a certain way and can be divided into two fundamental forms (Ryan \& Deci, 2001). Intrinsic motivation refers to the behaviour which is driven by internal rewards and consequences, whereas extrinsic motivation can be explained through a person's behaviour to achieve some external reward, such as grades or tokens. Both attitudes and motivations hold the potential to facilitate any learning process (Stiles, 1997). However, they can also yield detrimental effects and function as barriers if they are not fully understood.
Research suggests that students who are externally motivated and lack positive attitudes towards learning second languages give up more quickly than those who are intrinsically motivated (Bruce, 2006). This process is important to understand because it may offer explanations as to why a low percentage of Māori language learning occurs today, despite the vast array of opportunities available. In addition, these two forms of motivation are important predictors of achievement levels and help with identifying why some students enjoy and engage in particular activities (Grant \& Dweck, 2003). Numerous studies, such as Ryan and Deci (2001) and Davis, Bagozzi and Warshaw (1992), have shown that students who are intrinsically motivated are more likely to engage in work and be fully challenged and fulfilled compared to those who are motivated by external rewards. Consequently, it is important to understand the factors which influence intrinsic motivation in order for teachers and educators to promote this approach within the educational context of teaching te reo.

It is also important to understand that first language learning occurs without any significant motivation (Gardner \& Lambert, 1972). However, learning a second language in adulthood or at university is a completely separate process, both cognitively and socially (Rātima \& May, 2011). Consequently, understanding this dynamic interaction between the array of different motivational factors and attitudes held may facilitate the learning process and assist in understanding how language learning develops amongst adult learners. In addition, as the speakers of Indigenous languages such as te reo continue to decrease around the world, so do cultural resources and Indigenous identities. As Te Puni Kōkiri (2003) observe, "Ko te reo 
te mauri o te mana Māori” (p. 42, language is the essence of Māori power). Therefore, understanding attitudes and motivations of learning Indigenous languages that are endangered is of high value to the greater goal of language revitalisation.

There is a small set of studies focused on examining the attitudes and motivations of second language learners. One such study, which was conducted across England, Germany and the Netherlands by Bartram (2006), aimed to investigate the effects of attitudes on students' learning of an additional language. A total of 411 students were asked to fill out a survey which focused on measuring their attitudes and perceptions towards their parents. Bartram (2006) hypothesised that strong parental influences may shape children's orientations towards foreign language learning. The findings suggested that when parental attitudes are perceived as positive, this positivity is indeed mirrored in their children's orientations. Simply put, students whose parents held positive attitude towards language learning had a positive attitude towards language learning themselves.

A similar study by Oliver and Purdie (1998) surveyed 58 Australian children's attitudes and motivations towards their first and second languages. The researchers found that students' motivation towards language learning is influenced by the attitudes they hold about their first and second languages. Further, a vast array of influences, including parents, peers and teachers, can determine such attitudes. Oliver and Purdie (1998) also suggested children are similar to adults in that they are strongly influenced by those who are significant to them. They suggest these influences can play an important role in children's attitudes and motivations towards language learning.

\section{Attitudes in New Zealand}

A small number of studies have been conducted in New Zealand related to examining attitudes and learning languages. For example, one study by Barkhuizen, Knoch and Starks (2006) surveyed a sample of 920 high school students from a variety of different ethnic groups to examine attitudes and preferences towards learning languages. The researchers found that children's understandings of themselves and their own languages are significantly influenced in schools through contact with other children. Furthermore, the attitudes held by students play an important role when examining behaviour. The results indicated that the behaviours and attitudes of majority language speakers can be changed and play a significant part in language learning and maintenance.

In another study, McComish (2004) conducted a literature survey in New Zealand schools to establish motivations towards learning and teaching te reo. Like previous studies which found attitudes held by parents were influential and mirrored those of their children, McComish (2004) found that parents and families can also positively influence students' motivation towards learning te reo Māori. She further noted the need to understand the dynamic relationship between family members and students, as this may also help with reducing feelings of anxiety or negativity that many people have about learning another language.

Albury (2016) used a quantitative and qualitative survey to investigate how approximately 1,300 Indigenous and non-Indigenous youth aged between 18 and 24 years defined Māori language revitalisation from their own perspective and how they perceived the revitalisation processes and outcomes proposed in scholarship and local discourses. The results suggested that linguistic knowledge exists parallel to language attitudes, but also informs local policy ideas. Albury (2016) also found that literacy was an important component and played a significant role when considering revitalisation efforts. Furthermore, high Māori language proficiency was not a necessary component for increasing motivation. 
De Bres's (2010) research extended previous studies by examining attitudes and behaviours of non-Māori New Zealanders towards the Māori language, focusing on two behaviours relating to the use of Māori in New Zealand English, namely the use of Māori words and phrases and the pronunciation of Māori words. The methodology for the study involved administering questionnaires and collecting interview data. Eighty non-Māori New Zealanders at nine workplaces in Wellington participated in the study. The results showed evidence of negative attitudes towards the Māori language among some participants. However, a number of participants also held positive attitudes towards the Māori language. De Bres suggested that the research serves as an important reminder that, when talking of the attitudes of non-Māori in relation to the Māori language, we should have a diverse group of people in mind.

Most research in this area has examined only attitudes or motivations in isolation while ignoring whether there is a connection between the two. Further, surveys have been the main method of data extraction, which can fail to elicit a comprehensive understanding about students' attitudes and motivations (Keegan, Mato, \& Ruru, 2015). Attempting to rectify this situation, the government commissioned four telephone interviews, in 2000, 2003, 2006 and 2009 (Te Puni Kōkiri, 2010). The surveys measured knowledge, attitudes and general values about the Māori language among both Māori and non-Māori respondents. Each year, approximately 1,500 respondents provided an explanation of their attitudes and motivations towards the Māori language. The results have shown that Māori in general hold positive attitudes towards learning te reo. However, one important component which was verbalised was the lag between positive attitudes and reported participation and motivation by Māori in language and cultural events (Te Puni Kōkiri, 2003). Although family members and students may hold positive attitudes towards te reo, researchers inferred that this does not essentially mean behaviours and people's motivations will match.

While the studies described above show promising insights into individuals' attitudes and motivations towards learning te reo, none of them involved teacher trainees. This population is an important part of the system for change because, with the appropriate skills, teachers can help aid revitalisation efforts for te reo through engaging children in activities such as short phrases or games which are crucial for prompting the development of te reo within the classroom. Additionally, only a small set of studies have addressed both attitudes about and motivations for learning te reo. Focus groups have not been the chosen methodological approach despite their ability to allow participants to collectively generate different perspectives and compare their ideas with those of others. Clearly, more research is needed to investigate attitudes and motivations specifically with teacher trainees.

The present study was designed to extend the work on identifying attitudes towards and motivations for learning te reo in order to make revitalisation efforts more effective. The aim was to investigate teacher trainees' attitudes and motivations about an optional Māori language course in order to provide a thematic overview and guide future research on te reo revitalisation efforts. The researcher hypothesised that conversing with students and picking up on non-verbal cues may provide a clearer understanding of students' ideas. Attitudes and motivations were collected via an online qualitative questionnaire and a focus group, with an external member taking notes and a facilitator asking questions to determine what factors have influenced the students' desire to learn te reo. Lastly, as the current study followed Oliver and Purdie (1998) and used small sample sizes, each question was developed with the idea of being able to provide lengthy records in order to be coded into themes. 


\section{Method}

\section{Rationale and philosophical assumptions}

The research was designed as an interpretive case study that was analysed using qualitative methods. Online questionnaires were used to evaluate participants' attitudes and motivations to determine their levels of interest in choosing to participate in an optional te reo course outside of class time. A focus group interview was then used as second data collection method to allow participants to develop and express ideas they might not have thought about on their own (Corfman, 1995) The focus group was on participants' multiple perceptions and meanings of events in deciding to attend a te reo course that was available to all of the students in the paper. The interpretive paradigm underpinned the current research as it involved collecting information and drawing conclusions about events, and the data collected were interpreted to make meaning of the information shared.

\section{Ethical approval and informed consent}

Ethical approval was granted by the Human Ethics Committee at Victoria University of Wellington, New Zealand (Application \#0000024628). All participants were given both oral and written information about the study, and they were reassured that their anonymity and details of the research would be protected. Written informed consent was obtained from all the participants before the focus group discussion commenced.

\section{Participants}

Participants who were selected for the study attended the same undergraduate course in a teaching programme at Victoria University of Wellington. The only inclusion criterion for participating was that the student had to have signed up for a specific educational paper which had a separate and optional Māori language component that took place every Wednesday for 10 weeks. Three teacher trainees participated in the focus group discussion, all of whom regularly attended the optional Māori language session. Two of the participants had lived in New Zealand their entire life and had previous exposure to te reo learning in school. The other participant had recently moved to New Zealand, was taking an additional te reo Māori course in another institution, had English as a second language and could speak two other languages. Seven additional teacher trainees, for whom no background information was obtained, completed the online survey. De Bres's (2010) research suggested it is important to include a diverse number of participants. Accordingly, the online survey was designed to capture diversity without specifically recording personal details. The course supervisor notified the students about the study at the end of lectures and through course announcements. Survey links were also available to members of the class to complete at their leisure.

\section{Setting}

The focus group interview was conducted in a quiet room on the university campus. A semi-structured interview guide that included open-ended questions was used to facilitate the discussion. The questions were based on former interviews that had been conducted to examine the attitudes and motivations behind students' decisions to study particular languages (Djigunovic, 2012). The focus group lasted approximately 1 hour, until the topic was covered to the satisfaction of participants and the interviewer. The online survey was completed at the leisure of the participants.

\section{Design and procedure}

A question guide was followed during the focus group to ensure all of the topics important to 
the research question were covered and to allow additional issues to be raised. After an initial introduction, the questions which explored attitudes in the focus group included:

- Do you think learning te reo Māori is important? Why or why not?

- What do you think has influenced your views about learning te reo Māori the most?

- Have your views about learning te reo Māori changed while participating in Kōrero Mai classes (the optional beginner level te reo Māori course)? Why or why not?

The questions which explored motivations included:

- What are the key factors that motivate you to learn te reo Māori?

- How are you developing your understanding of te reo Māori outside of your Kōrero Mai lessons?

- How do you plan on furthering your understanding of te reo Māori after the completion of this course?

At the end of the discussion, a koha was given to the students to thank them for giving up their time.

The online questionnaire measured attitudes and motivations on a five-point scale and consisted of the following questions:

- How important is learning te reo Māori to you?

- What has influenced your views on learning te reo Māori the most?

- Have your views about te reo Māori changed while participating in Kōrero Mai?

- Do you envision te reo being useful in the near future?

- What are the key factors that motivate you to learn te reo Māori?
- What do you do outside of your Kōrero Mai classes to develop your understanding of the language?

- How likely are you to extend your knowledge after this course?

- Does learning te reo provide an insight into the Māori culture?

- Do you use many resources to help with your understanding of te reo?

- How difficult has it been to learn te reo?

A focus group was chosen as a second research method as it seemed to best align with the objectives of this research, which aimed to explore students' attitudes and motivations for learning te reo. Also, focus groups encourage participants to collectively develop ideas and explore specific issues based on their actual experiences related to te reo (De Bres, 2010). Focusing on the participants' narratives enables an exploration of ideas which can be developed into meaningful statements. The dynamic group process may also yield results that bear resemblance to the general population's attitudes and motivations of learning te reo Māori. This approach also aligned with a decolonising methodological approach as it plays a significant role in the assertion of Indigenous people's rights and sovereignty (Rātima \& May, 2011).

The focus group lasted around an hour, was semi-structured and contained questions about attitudes and motivations towards learning te reo. Prompts were used throughout the discussion to further clarify the participants' narratives and to ensure that a clear understanding between participants and researcher was achieved. The facilitator recorded the discussion with key phrases and words on a notepad and the postgraduate student recorded the discussion on the laptop.

Questionnaires were also available to be completed online from a link provided on Blackboard. Seven of the students completed the questionnaires and the results were printed off to supplement the focus group discussion. The data from the focus group and online surveys 
were analysed by the researcher and five major themes were identified and developed.

\section{Results}

The analysis identified five distinct but related themes that were commonly found across participants: (a) importance of engaging in culture alongside the language, (b) consistent learning throughout school and college, (c) motivation can be enhanced through everyday use of the language with friends and whānau, (d) large expenditure of time and effort is required to access Māori speaking communities and (e) mismatch between attitudes towards Māori language and behaviours.

\section{Importance of engaging in culture alongside the language}

All three focus group participants and six out of the seven survey responses identified the need to learn tikanga alongside te reo. This is because there is an inherent connection between the two which results in the language being ingrained and expressed in the cultural values and practices (Rātima \& May, 2011). Furthermore, the two are inseparable: developing one results in a deeper knowledge and understanding of the other. Subsequently, students can develop their language skills and cultural competency through both of these two fundamental aspects (Te Puni Kōkiri, 2003). Participants reflected this by stating that without learning tikanga the language would not be as easy to learn and would be less rich and complex.

I remember learning some stories and songs at school which were cool and helped with my learning of the language, and then some concepts in PE that also made me think this language is pretty complex when you think about it. (Participant 1)

Participants mentioned that culture was even more important to them than speaking the language. They found that it may be more helpful in the classroom to understand Māori values and expectations in order to relate to their students. These comments align with the work of Albury (2016), who suggests that language is not intrinsic to being Māori.

I learnt te reo so I could understand more about the ways in which Māori practise their beliefs rather than actually speaking the language. It makes the language richer when you learn the culture with it. Otherwise there is kind of like this whole missing. (Participant 3)

I think if I can understand some of the children's expectations than I may be able to help them in my class more. (Participant 2)

Understanding the impact of tikanga and how it shapes the way people think and behave is crucial not only for developing the language but also for preserving it for the next generation (Rātima \& May, 2011). Tikanga, which embodies customs and traditions and ensures Māori maintain their distinguishable and unique features, plays a pivotal role in the learning process of te reo and should not be ignored (Cram, 2009). Therefore, if tikanga and language are learnt together in the classroom, this may not only help assist students' language acquisition, but also "normalise" reo learning by breaking down any stigmas or barriers attached. One practical solution to this could be teaching both aspects together in early education settings while continuing the learning process throughout students' schooling to maximise long-term benefits.

\section{Consistent learning throughout school and college}

According to the experiences of the participants who completed the focus group, learning a second language through minimal sessions for extended amounts of time was not helpful for their retrieval and understanding of the 
language. This is consistent with research which shows the distribution of learning can have significant effects on the learning and retention of information (Keppel, 1967). According to Baddeley and Longman (1978), if spaced learning and study over time is promoted over massed practice of material, students are more likely to gain a deeper understanding, and there is more chance of information being coded into long-term memory. The two participants in the focus group who were educated in New Zealand schools both mentioned how te reo was taught to them in chunks with no consistent learning over time. Subsequently, both participants were unable to remember many words or sentences as a result of this learning strategy.

I remember learning a whole heap of te reo when I had one specific teacher and then after that nothing and now it comes back to me slowly. (Participant 1)

Year 5 and 6 were awesome because my teacher taught us a word a day, but after that nothing and now I have forgotten all of it, which sucks. (Participant 3)

Both students mentioned the difficulty of being able to retrieve information they had learnt when they were younger. This effect can be due to learning in massed practice, which may demonstrate short-term benefits for learning and retrieval. The studies above do not support this approach for deep understanding and long-term benefits. This effect was also alluded to by the participant who learnt English as a second language.

I remember learning English a couple of days a week and nothing really stuck. It wasn't until I learnt it every day and was exposed to English news and other sources that I started being able to speak it. (Participant 2)

Based on this response, spaced practice in addition to high levels of exposure to the target language helped promote a fostering immersion environment for learning. This is consistent with the literature that shows low exposure to the target language may have ceiling effects compared with high rates of exposure, which demonstrate long-term retrieval and comprehensive benefits (Rifkin, 2005). Subsequently, learning methods and higher rates of exposure from various sources such as news, books or apps may assist in promoting rich language learning environments necessary for the promotion of te reo (Ryan \& Deci, 2001). With more exposure, consistent learning and possibly more resources to choose from, students' motivation may also be increased towards studying te reo.

\section{Motivation can be enhanced through everyday use of the language with friends and whānau}

Most respondents who completed the online survey and most participants in the focus group agreed their motivation was increased by their parents' encouragement. Support from family members was also helpful for reducing negative feelings and stigmas attached to practising reo in society.

I often practise a few words around the home because sometimes I feel weird saying it in public ... people may think I'm trying too hard. (Participant 1)

There is an email list I subscribe to and get a word a day which is cool and easy to learn and use with family members. (Participant 3 )

Friends were also driving motivational forces, with five out of the seven survey respondents suggesting peers were the biggest influences in their learning. Furthermore, one student mentioned how important Skyping with her friend was for learning te reo: "My Skype session with my friend really motivates me because I can see the practical use in the language and my friend's a really good teacher too" (Participant 3). 
Opportunities to converse with friends and family members allowed students to take risks and experiment with the language. They could engage in different topics and discussions. which helped them make sense of the language.

These two factors should not be underestimated or ignored in the process of language learning. Therefore, although Skyping friends may not always be possible for students, the ability to engage in te reo as frequently as possible with trusted and close individuals is crucial. This may also help to lower linguistic anxiety that often arises in minority contexts (Sevinç \& Dewaele, 2018). In addition, it is important to promote this approach to develop consistent connections whereby reo can be easily spoken and practised. Forming trusted connections with others may promote the usage and skills of te reo Māori learning.

\section{Large expenditure of time and effort is required to access Māori speaking communities}

All participants reported difficulty in accessing environments where they could kōrero with other individuals who spoke te reo. This is partially due the relatively small population of te reo speakers, who tend to be located in clusters in particular regions in Aotearoa ( $\mathrm{Te}$ Puni Kōkiri, 2003). This makes it difficultespecially for those who are busy with other courses-for learners to engage in rich language speaking communities. As Participant 3 commented, "I rely on my Skype time with my friend up north because I don't know too many people around here who speak it and, I also feel embarrassed sometimes if I speak it in Wellington."

Becoming proficient in any language requires effort on the part of the learner to engage with the community who speak it. However, if too much effort is required then there is more possibility that the learner will give up (Nation, 2014). This idea was evident throughout the focus group.
We have such little time to practise te reo so I often find myself doing other things instead. (Participant 1)

I think it is useful, but can't see myself continuing to learn it in the future because there is not many people I can just go and have a conversation with like French or Spanish. (Participant 2)

Although students were generally optimistic about the continuation of learning te reo, all three students in the focus group mentioned the problematic issue of finding more environments to practise after the course finished. As a result, they thought they would be unlikely to continue the language learning process.

I might try to keep learning it has been helpful, but it's going to be hard finding a place that actually teaches you rather than you learning off an app. (Participant 1)

I'm not going to continue, just too much of a time constraint to find a place. (Participant 2)

Students saw the Kōrero Mai sessions as a good starter, similar to learning the language at school. However, if they are to take their understanding to the next level, there needs to be easier access to rich language environments (Leffel, Suskind, \& Suskind, 2013). One possible and practical solution would be to encourage more people who are fluent in te reo to become teachers. This would subsequently promote more fostering language environments throughout New Zealand and may give those individuals who hold positive attitudes towards learning te reo easier pathways to the next level of language development.

\section{Mismatch between attitudes towards Māori language and behaviours}

All participants who completed the survey held positive attitudes towards learning te reo and 
identified benefits in learning the language. However, this was not reflected in their future plans regarding the study of te reo. This mismatch was further emphasised in the focus group, in which participants identified the importance of the language but also did not want to continue studying it: "Te reo is one of our national languages and of course it is important . . . Although I don't think I will continue to learn it after the course" (Participant 1).

It is possible the numerous initiatives and strategies designed to promote te reo have raised people's awareness of the importance of the language and the impact it has on New Zealand's national identity. These initiatives may be positively impacting how the language is perceived by both Māori and non-Māori, but they are not necessarily assisting in the learning of the language (Christensen, 1997). Another factor which could be contributing to this mismatch between attitudes and behaviours is the available time teacher trainees have to learn and teach te reo.

To be honest, with my student loan and the amount of study I have to do, if I had extra time I would be working to get some money rather than learning te reo. (Participant 3)

We are here at university all of the time and it is just too hard to fit in more language. Plus, there are so many things to teach the children nowadays it would be hard to fit another component into the curriculum. (Participant 1)

Most students face the problem of time constraints and this was evident during the focus group. Te reo is simply not valued enough for these students to actively seek out resources and commit to the language learning process, when they are already faced with other challenges in managing their workload. However, because te reo is not compulsory in New Zealand schools it is understandable that teachers will not feel the need to commit many hours to learning it. Making the language compulsory in schools would increase teacher trainees' motivation to learn the necessary skills to teach reo. This strategy could also function as a positive motivational force to drive teachers' and students' desire to speak the language as it would be recognised as an important aspect of the education curriculum. This point was emphasised by the focus group participant who completed their schooling years abroad: "Overseas where I learnt English, it was compulsory, and everyone had to learn it. It's a lot different here where [te reo is] a choice and not many people seem to care" (Participant 2).

Another concern students had was pushing other subjects out of focus in order to fit te reo into the curriculum, which is already full of subjects that need to be taught: "To be honest, what would we take out and when would we be able to teach it in school with heaps of subjects to teach?" (Participant 1). However, as previously stated, deep understanding and long-term retrieval do not require vigorous hours of work with massed practice strategies. Research has demonstrated it simply requires constant work over long periods of time in addition to high levels of exposure. It may therefore be possible to add te reo into an existing module or subject rather than creating an additional unit to teach (Buchsbaum \& D'Esposito, 2013). This practical strategy could take the form of learning a word every day, simple grammar rules, a whakatauki or learning how to sing a waiata together as a class. Minimal amounts of time would be spent by teachers and educators with such a strategy, which could help maximise the opportunities for revitalisation efforts towards te reo.

\section{Discussion}

This research project explored teacher trainees' attitudes and motivations towards learning te reo. These two factors were chosen because understanding them will benefit future revitalisation efforts for te reo and help identify the 
barriers teacher trainees are faced with when it comes to learning the language. It is hoped that the following recommendations will benefit not only the teacher trainees themselves and the community where the research was based but also similar learning environments throughout Aotearoa that are focused on preserving te reo.

This research took a qualitative approach and employed a combination of an online survey and a focus group discussion. This research design had the advantage of generating insightful ideas and insight into what teacher trainees' attitudes and motivations were towards learning te reo. It was evident that the discussion process itself questioned the thinking of the focus group participants. Together the focus group and surveys were successful in providing a deeper understanding into trainee teachers' attitudes and motivations.

It is important to consider the social and political context in which this research is situated. As May (2011) highlights, any analysis of bilingualism must always account for the wider social and political context, which includes recognising the varying status ascribed to te reo and its minority status in New Zealand. This can help us understand why a minority language such as te reo is not valued as much as its dominant counterpart. Despite recognising the educational or linguistic benefits of learning te reo, the teacher trainees in this study tended to ascribe to the idea that the majority language, English, is more socially and politically advantageous in the New Zealand context.

Three themes were consistent with the literature: (a) the importance of learning tikanga with te reo, (c) motivation can be enhanced through everyday use of the language with friends and whānau and (e) there is a mismatch between attitudes towards the Māori language and behaviours (Keegan et al., 2015; McComish, 2004; Oliver \& Purdie, 1998). However, the other themes-(b) consistent learning throughout school and college and (d) the large expenditure of time and effort required to access Māori speaking communities-provide additional evidence for the importance of understanding attitudes and motivations towards learning te reo. The significance of the current study, therefore, rests not solely on the novelty of these two new themes but also on the fact that the findings confirm and extend existing knowledge on the significant role attitudes and motivations play in fostering the future development of te reo (King, 2001).

\section{Implications}

\section{Tikanga and consistent learning}

After identifying the important role tikanga has in the development of the language, it is fundamental to include this aspect in any form of language learning provided by educators. Due to the nature of this relationship, it is also vitally important to start learning the language and culture together as early as possible while continuing its development over longer periods of time. The findings of this research also suggest the need to start language development early. This is crucial not only for language acquisition but also for promoting motivations and attitudes towards learning (King, 2001). One or two words a day over a long period of time, in addition to providing grammar and tikanga knowledge, would help build up students' vocabulary and assist them in making sense of the Māori world. Further, it may alleviate some of the cognitive and social pressures on adults when learning a second language later on in life (Wong Fillmore, 1991). This aligns with the findings of May (2011), who emphasises the need to identify the school context in which bilingual programmes are situated. This is because the school environment provides an ideal opportunity for learning to take place. Therefore, incorporating te reo Māori vocabulary into the school curriculum is one recommendation for revitalisation efforts. 
Friends, whānau and the importance of te reo

Friends and family members are influential people in the learning process and their support needs to be encouraged by educators. As demonstrated by the participants in the focus group, sharing knowledge with others and experimenting with the language allows students to explore the language for themselves. When teaching te reo, it may be beneficial to assign students with peers to practise with to help facilitate their development of the language. Further, family members could also take on the responsibility to provide opportunities for students to practise and develop their skills. However, as it is difficult to access Māori speaking communities it may be hard to practise with fluent speakers of the language. Subsequently, the need to provide funding for te reo teaching and ensure it is appealing to do so, is crucial for the survival of the language. In addition, both the surveys and the focus group alluded to the importance participants put on the language, yet many participants were not willing to continue learning. Fundamentally, this comes down to the need and importance placed on it by society. If it is not a compulsory component of the education curriculum, there is very little which can be done to encourage teachers to learn and incorporate tikanga and te reo into the classroom. Therefore, more government support is needed to raise the value and importance of the language for its survival.

\section{Limitations and future directions}

This study, like all research endeavours, has its limitations. It is possible that responses generated from the focus group were influenced by social desirability and/or other psychosocial factors. For example, participants may have stated they held positive attitudes towards the language because they felt this was socially desirable. However, this possible limitation is inherent to focus group research methods, and although significant attempts were made to reduce biased responses through creating a safe environment and using non-threatening, open-ended questions, biases may have still existed. Another limitation of the focus group was not accessing participants with a variety of sociodemographic characteristics. Two of the participants were of similar age and had similar life experiences, and all participants were of the same gender. Therefore, the teacher trainees' attitudes and motivations were not representative of the general population.

Further, the small sample size-due to the difficult nature of recruiting full-time teacher trainees-along with the qualitative design of the study, means causal inferences cannot be made from the findings. Future research that employs quantitative or mixed methods approaches to further explore the various aspects of teacher trainees' attitudes and motivations may identify further revitalisation recommendations in order to make more robust and definitive statements regarding the future. Investigators with more time and resources available should aim to recruit larger and more demographically diverse teacher trainees from around New Zealand in order to gain a more complete understanding of their views on te reo.

\section{Conclusion}

The study findings confirm what is known about the role of attitudes and motivations on learning te reo. They also extend previous research by being drawn from an under-researched population-teacher trainees-who will be in a position to educate and potentially foster language-rich environments for developing te reo. What emerges is a complex picture that includes the importance of understanding culture, ensuring there is consistent learning, including friends and whānau in the learning process, providing more opportunities to engage in the language, and raising the value and importance of the language. These findings 
can inform future revitalisation efforts, promote learning and help address some of the barriers faced by teacher trainees.

What is clear from the findings of this study is that due to the scarce number of competent te reo teachers and the lack of importance placed on learning te reo in schools, there is no instrumental need for teacher trainees to learn one of New Zealand's official languages. Further, the findings suggest that unless te reo is compulsory in schools and there is easier access to environments for practice, this teacher population, who can potentially facilitate children's learning and understanding of the language and culture, will struggle to increase in future years. Given the little attention paid to, and limited understanding of, teacher trainees' attitudes and motivations towards learning te reo, additional investigation is warranted. In particular, attention should be paid to teachers' individual abilities to competently speak and teach te reo in the classroom.

The current study's findings, combined with those from existing research, are a starting point for an improved understanding of what is required to help increase the number of New Zealanders able to speak te reo Māori. Teacher trainees equipped with the skills to proficiently speak and educate children in te reo may significantly boost revitalisation efforts. Continued investigation of teacher trainees and their attitudes and motivations will be necessary to answer many of the questions that remain.

\section{Glossary}

Aotearoa Māori name for New Zealand; lit. "land of the long white cloud"

koha gift

kōrero talk

te reo Māori the Māori language

tikanga Māori customary practices and behaviours

waiata song

whakataukī proverb

whānau extended family grouping 


\section{References}

Albury, N. (2016). Defining Māori language revitalisation: A project in folk linguistics. Journal of Sociolinguistics, 20(3), 287-311. http://doi.org/ f87d9c

Baddeley, A., \& Longman, D. (1978). The influence of length and frequency of training session on the rate of learning to type. Ergonomics, 21(8), 627-635. http://doi.org/c787h9

Barkhuizen, G., Knoch, U., \& Starks, D. (2006). Language practices, preferences and policies: Contrasting views of Pakeha, Maori, Pasifika and Asian students. Journal of Multilingual and Multicultural Development, 27(5), 375-391. http://doi.org/czjk75

Bartram, B. (2006). An examination of perceptions of parental influence on attitudes to language learning. Educational Research, 48(2), 211-221. http://doi.org/cr482d

Bruce, I. (2006). English in the mainstream: Heritage language or lingua franca? English in Aotearoa, $59,17-21$.

Buchsbaum, B., \& D’Esposito, M. (2013). Working memory. In K. N. Ochsner \& S. Kosslyn (Eds), The Oxford handbook of cognitive neuroscience, volume 1: Core topics [Online]. Oxford, UK: Oxford University Press. http://doi.org/cs5z

Christensen, I. (1997). Maori language in the Manawatu Whanganui region. He Pukenga Korero: A Journal of Maori Studies, 2(2), 24-30.

Corfman, K. (1995). The importance of member homogeneity to focus group quality. Advances in Consumer Research, 22, 354-359.

Cram, F. (2009). Maintaining Indigenous voices. In D. M. Mertens \& P. E. Ginsberg (Eds), The handbook of social research ethics (pp. 308-322). Thousand Oaks, CA: Sage. http://doi.org/ctz3

Davis, F., Bagozzi, R., \& Warshaw, P. (1992). Extrinsic and intrinsic motivation to use computers in the workplace. Journal of Applied Social Psychology, 22(14), 1111-1132. http://doi.org/frfk5w

De Bres, J. (2010). Attitudes of non-Māori New Zealanders towards the use of the Māori in New Zealand English. New Zealand English Journal, 1-13.

Djigunovic, J. M. (2012). Attitudes and motivation in early foreign language learning. Center for Educational Policy Studies Journal, 2(3), 55-74.

Eagly, A. H., \& Chaiken, S. (1993). The psychology of attitudes. Orlando, FL: Harcourt Brace.
Gardner, R., \& Lambert, W. (1972). Attitudes and motivation in second-language learning. Rowley, MA: Newbury House.

Grant, H., \& Dweck, C. S. (2003). Clarifying achievement goals and their impact. Journal of Personality and Social Psychology, 85(3), 541-553. http://doi.org/dfq2p4

Keegan, T. T. A. G., Mato, P. J., \& Ruru, S. (2015). Using Twitter in an Indigenous language: An analysis of te reo Māori tweets. AlterNative, 11(1), 59-75. http://doi.org/cs53

Keppel, G. (1967). A reconsideration of the extinctionrecovery theory. Journal of Verbal Learning and Verbal Behavior, 6(4), 476-486. http://doi.org/ dpr3c2

King, K. A. (2001). Language revitalization processes and prospects: Quichua in the Ecuadorian Andes. Clevedon, UK: Multilingual Matters.

Leffel, K., Suskind, E., \& Suskind, D. (2013). Project ASPIRE: Cultivating rich early language environments for all children. Volta Voices, 20(3), 30-33.

May, S. (2011). Language and minority rights: Ethnicity, nationalism and the politics of language (2nd ed.). New York, NY: Routledge.

McComish, J. (2004). Literature survey on second language learning and teaching as it relates to te reo Māori as a subject in schools. Auckland, New Zealand: Learning Media.

Nation, K. (2014). Lexical learning and lexical processing in children with developmental language impairments. Philosophical Transactions of the Royal Society B: Biological Sciences, 369(1634), 1-10. http://doi.org/ctz2

Oliver, R., \& Purdie, N. (1998). The attitudes of bilingual children to their languages. Journal of Multilingual and Multicultural Development, 19(3), 199-211. http://doi.org/fw4fcj

Rātima, M. T., \& May, S. (2011). MAI Review, 1-25. Retrieved from from http://www.review.mai. ac.nz/index.php/MR/article/view/412/594

Rifkin, B. (2005). A ceiling effect in traditional classroom foreign language instruction. Modern Language Journal, 89, 3-18. http://doi.org/ $\mathrm{d} 4 \mathrm{ps} 4 \mathrm{~d}$

Ryan, R. M., \& Deci, E. L. (2001). To be happy or to be self-fulfilled: A review of research on hedonic and eudaimonic well-being. Annual Review of Psychology, 52, 141-166.

Sevinç, Y., \& Dewaele, J. (2018). Heritage language anxiety and majority language anxiety among Turkish immigrants in the Netherlands. 
International Journal of Bilingualism, 22(2), Te Puni Kōkiri. (2010). Attitudes toward the Māori 159-179. http://doi.org/cs54

Statistics New Zealand. (2013). New Zealand official language. Retrieved from https://www.tpk.govt. nz/en/a-matou-mohiotanga/language/summaryyearbook 2008. North Shore City, New Zealand: David Bateman. sheet-of-the-2009-maori-language-attitudes/ online/3

Stiles, D. (1997). Four successful Indigenous language programs. In J. Reyhner, (Ed.), Teaching Indigenous languages (pp. 248-262). Flagstaff, AZ: Northern Arizona University.

Te Puni Kōkiri. (2003). Te rautaki reo Māori: The Māori language strategy. Wellington, New Zealand: Author.

Wong Fillmore, L. (1991). When learning a second language means losing the first. Early Childhood Research Quarterly, (3), 323-346. http://doi. org/c7zcpm 